\title{
Non-Invasive Second Harmonic Generation (SHG) in Macroscopy (MacroSHG) as Bio-Diagnosis to Image Collagen Network Organization in Extracellular Matrix
}

\author{
Dominique Dumas ${ }^{1,2 *}$, Elisabeth Werkmeister ${ }^{3}$, Sebastien Hupont ${ }^{2}$, Céline Huselstein ${ }^{1}$, \\ Natalia De Isla1, Marthe Rousseau', Patrick Menu', Didier Mainard1,2 \\ ${ }^{1}$ Universite de Lorraine, Physiopathologie, Pharmacologie et Ingénierie Articulaires (IMOPA, CNRS 7365), \\ Nancy, France \\ ${ }^{2}$ Ingénierie Moléculaire et Thérapeutique (CNRS FR 3209), Plate Forme d'Imagerie Cellulaire et Tissulaire \\ PTIBC-IBISA NANCY, Nancy, France \\ ${ }^{3}$ Plate-Forme Microscopie-Imagerie-Cytométrie du campus Pasteur Lille (MICPaL), IFR 142 Institut Pasteur de \\ Lille, Institut de Biologie de Lille, Lille, France \\ Email: ${ }^{*}$ dominique.dumas@univ-lorraine.fr
}

Received 22 April 2014; revised 28 May 2014; accepted 10 June 2014

Copyright (C) 2014 by authors and Scientific Research Publishing Inc.

This work is licensed under the Creative Commons Attribution International License (CC BY).

http://creativecommons.org/licenses/by/4.0/

(c) (i) Open Access

\section{Abstract}

In this work, we have considered a new multimodality imaging for macroscopy based on Second Harmonic Generation (SHG) method to monitor invasivelessly the matrix collagen. As the triple helicoidally structure of collagen molecules appearing as not centrosymetric, very organized and spatially oriented, collagen fibrils give rise to a very strong SHG signal and can be imaged without any exogenous dye. To integrate a multidimensional scale with a large field of view (non-sliced samples), we have adapted and validated an instrumental coupling between a two photon excitation laser and a macroscope to collect cartography of SHG signal. We introduced an index (F-SHG) based on decay time response measured by TCSPC for respectively Fluorescence (F) and Second Harmonic Generation (SHG) values. For various sample where protein collagen is the major component of extracellular matrix (vessel, skin, carotide vessel, rat femoral head cartilage, mouse tumor, human wharton's jelly and rat tendon) or not (nacre), we compared the index distribution obtained with MacroSHG. In this work, we showed for the first time that multiscale large field imaging (Macroscopy) combined to Multimodality approaches (SHG-TCSPC) could be an innovative and non-invasive technique to detect and identify some biological interest molecules (collagen) in biomedical topics.

\footnotetext{
${ }^{*}$ Corresponding author.
} 


\section{Keywords}

\section{Multiphoton Macroscopy, Second Harmonic Generation, Collagen, Extracellular Matrix}

\section{Introduction}

To appreciate a high level of complexity in 3D organization of collagen network into biological matrix, optical microscopy, as non-destructive imaging technique, has a number of strong advantages over alternative imaging modalities (MRI, CT-scan, X-ray, arthro-scan, OCT, etc.). Conventional optical techniques for collagen detection, like histology (red Sirius) and fluorescence (specific antibody), remained a reference for structural characterization of extracellular matrix (ECM). Some contrast agents must be added to reveal collagen network (organisation, denaturation, orientation) as structure of interest before observation in white light or fluorescence microscopy. For instance, the red Sirius as staining agent is specific of fibrillar collagens in polarized light (Sweat, 1964) and is sensitive to the alignment of the molecules of collagen (red color for the extracellular matrix and yellow color for some other structures). This technique is well appreciated to estimate the topography of network of collagen in ECM but it is not dedicated to quantify the amount of fibrillar collagen molecules. Moreover, these techniques based on histological or immunohistochemical staining are invasive by introduction of exogenous dye (stain or antibody).

Major limitations due to restricted analysis on matrix in biological tissue (thick, opaque and scattering) have given rise to the development of other techniques to detect collagen molecules based on near infrared illumination as two-photon excitation microscopy (TPE) and Second Harmonic Generation (SHG). SHG is a non-linear light scattering effect that is restricted to high light intensities and very specific structure like cell membrane components, microtubules, or collagen fibers [1]. As processes involved in SHG are intrinsic properties of the constituent molecules (very organized triple helicoidally structure of collagen molecules, no centrosymetry and spatially oriented) non-linear microscopy allow submicron resolution images without the need for sectioning or staining with dye [2]. Moreover, as SHG is based on light scattering and not on fluorescence, photo-bleaching and toxicity are significantly reduced. As shown previously [3], the emission of quasi-instantaneously SHG photon diffused can be collected with a time correlating single photon (TCSPC) workstation originally dedicated to FLIM (Fluorescence Lifetime Imaging). The photon which is provided from SHG signal can be contrasted against a fluorescence intrinsic signal as encountered in cartilage tissue and immunolabelling staining. Only a very fast temporal decay method (TCSPC for Time-Correlated Single Photon Counting) can give a SHG signal (TCSPC-SHG) and it will not be the case with Frequency method (Phase/Modulation) usually used for FLIM. In TCSPC-SHG microscopy, the limitations due to photobleaching and phototoxicity in biological samples are overcome. This multimodality technique could be considered as a precise tool for biodiagnosis to detect in safety conditions collagen synthetized by cells seeded in scaffolds.

But this new approach in near-infrared SHG microscopy may not allow for sufficient tissue penetration to observe with a large field of viewing an organized network collagen. To avoid cutting of samples and collecting information on large dimension specimen, optical parameters linked to recent optical development in the field of macroscopy technique could be of interest in tissue imaging. In more, in vivo imaging capabilities allow for the capture of images that were previously not possible with traditional stereoscopic microscopes. Macroscope enables high-definition confocal image acquisition during macro observation and offers a combination of low and high magnification objective lenses and a scanning zoom function, enabling continuous imaging from macro to micro. To integrate a multidimensional scale with a large field of view and allow for analyzing as closed as possible of physiological conditions (non-sliced samples, sterile, non-invasive), we adapted and validated for the first time a coupling between a macroscope and a two photon excitation laser to realize a MacroMultiphoton dedicated to SHG signal (MacroSHG).

In this work, we proposed a study based on new SHG imaging contrast in macroscopy to detect "collagen index" from various tissues (nacre, vessel, skin, carotide vessel, rat femoral head cartilage, mouse tumor, human wharton's jelly and rat tendon). Here we integrated multiphotonmultiscale and multimodalities on macroscopy to detect collagen network. We showed that multiscale detection of SHG signal specific to collagen network is a non-invasive and potential technology in tomography to characterize the organized matrix. 


\section{Materials and Methods}

\subsection{Biological Samples}

Tendon and skin. A male rat, six weeks of age was sacrified whole Achilles tendon (tendocalcaneo) was harvested. Tendon was exposed in its full length after removal of the skin from the hind legs. For skin tissue sections, we excised a $5 \times 5 \mathrm{~mm}$ tissue.

Tumor and carotide vessel. Mice were used at seven weeks of age to generate tumours by subcutaneous injection of EMT6 cells into the right flank and tumour-bearing animals were sacrificed. Tumours and carotide vessel were removed, were rinsed with $\mathrm{NaCl} 0.9 \%$ and immersed in phosphate buffer saline solution at room temperature.

Nacre. Nacre sample was taken from the shell of the pearl oyster Pinctadamargaritifera (French Polynesia).

Cartilage. Articular cartilage was obtained from preserved areas of femoral condyles of patients (with informed consent) undergoing arthroplasty at the Department of Orthopaedic Surgery (University Hospital, Nancy, France). Cartilage was separated from the subchon-dral bone using a scalpel. Cylindrical explants (5 mm in diameter) were harvested using a sterile biopsy punch.

Wharton's jelly. It was obtained from human umbilical cords collected from full-term birth (with informed consent).

\subsection{Macro Multiphoton}

For Two photon Excitation system, a femtosecond oscillator (Mira 900F from 600 to $1100 \mathrm{~nm}$, Coherent), pumped with a solid laser (Verdi $8 \mathrm{~W}$, Coherent) was used to generate some ultra-short infrared pulses (120 fs, $76 \mathrm{MHz}$ ) at $\lambda=800 \mathrm{~nm}$. An EOM (Electro Optical Modulator LM 0202 P 5 W-n²1181, EO-Crystal KD*P $3 \times$ $3 \mathrm{~mm}^{2}, 5 \mathrm{~W}, 660$ - $1050 \mathrm{~nm}$, transmission 90.1\%, ratio 1/254) enabled to control the power of excitation (2.5 nJ per pulse at $76 \mathrm{MHz}$ ). With a scan head specially delivered for NLO (CLSM TCS SP2-AOBS, Leica Microsystems Manheim, Germany), Two photon Excitation Macroscope (MacroMultiphoton (patent WO 2012160312 A3)) [4] was realized by coupling the non-linear optical source (MiraF900) to macroscope (MacroFluoTM Z6 APO A, Leica Microsystems Manheim, Germany). The laser beam was focalized to the samples through a dry objective showing $\times 5$ magnification $(\mathrm{NA}=0.5$ ) and infra-red beam analyzer (Imaging French Network, RTmfm, MRCT, CNRS) used to calibrate optical alignment.

\subsection{TCSPC-SHG Imaging and F-SHG Ratio}

Decay times were measured in the backward direction by a time-correlated single photon counting workstation (SPC-730 TCSPC Imaging module, Becker \& Hickl, Berlin) coupled to scan-head of Macro Multiphoton. A signal coming from the scan controller of the microscope enabled to trace decay curves for each pixel of an image $(128 \times 128)$. The signal has to be deconvoluted with calculated instrumental response function and analysed for fitting with SPC Image software (Becker \& Hickl, Berlin). The time slop of the decay is usually a sum of different exponential decays and an index (F-SHG ratio) was described as the ratio between the long lifetime (intrinsic fluorescence relative to autofluorescence) and very fast lifetime (SHG due to collagen) which is obtained by two exponential components fitting. The SHG collagen exhibited instrument-limited response in the time-domain data with a full-width half maximum (FWHM) of 220 ps \pm 100 ps. Each fluorescent signal presents a characteristic lifetime decay signature ( $>500$ ps for tau2 component) and can therefore be discriminated and separated from the quasi-instantaneously SHG signal (220 \pm 100 ps for tau 1 first component). Autofluorescence signal exhibited a longer lifetime tail in their response due to fluorescence ( $2400 \mathrm{ps})$. These data indicated appreciable difference due to the presence of collagen in the integrated area of the transient response. When mean decay values measured for each pixel were coded as a colour LUTaccording to F/SHG index values, fluorescence signal was contrasted to SHG signal (see color code below).

\section{Results}

Under multiphoton excitation, macroscopygave access directly to visualization and comparizon between several samples containing or not high content of collagen (nacre, vessel, skin, carotide vessel, rat femoral head cartilage, mouse tumor, human wharton's jelly and rat tendon). Here, we present fluorescence and SHG images with 
a large field of view (about $25 \mathrm{~mm} \times 20 \mathrm{~mm}$ ) obtained by MacroSHG. The amplitude of SHG signal in sample is linked to presence or not of collagen. TCSPC-SHG data for extracellular matrix in various tissues (nacre, vessel, skin, carotide vessel, rat femoral head cartilage, mouse tumor, human wharton's jelly and rat tendon) are presented in Figure 1. Details given by multiphotonmacroscopy showed collagen fibers constitutive to network and fluorescence due to matrix components (Figure 1, columns A and B).

\begin{tabular}{|c|c|c|c|}
\hline & A. Intensity Image & B. Ratio lifetime Image & C. Ratio lifetime distribution \\
\hline Nacre & & & \\
\hline \multicolumn{4}{|l|}{ Rat skin } \\
\hline \multicolumn{4}{|c|}{ Rabbit carotid vessel } \\
\hline \multicolumn{4}{|c|}{ Cartilage from femoral head } \\
\hline \multicolumn{4}{|l|}{ Tumor } \\
\hline \multicolumn{4}{|c|}{ Wharton's jelly } \\
\hline \multicolumn{4}{|l|}{ Tendon } \\
\hline & & & 10 \\
\hline
\end{tabular}

Figure 1. Various biological tissues containg collagen in extracellular matrix (nacre, skin, vessel, cartilage, tumor, wharton's jelly, tendon) analysed in TCSPC-SHG macroscopy to extract F-SHG index. Scale: $1 \mathrm{~cm}$ bar for $1 \mathrm{~mm}$. 
For various tissues (vessel, skin, carotide vessel, rat femoral head cartilage, mouse tumor, human wharton's jelly and rat tendon) and mineral component (nacre), challenge was, using a prototype of MacroMultiphoton, to distinguish changes providing from fluorescence (F) signal and from Second Harmonic Generation (SHG) signal. In relation to matrix components and cellular properties, fluorescence collected was obtained from tissue autofluorescence signal. In the absence of exogenous dye, SHG signal is specific to a fibrillar arrangement of collagen molecules in extracellular matrix. SHG signal diffused was quasi-instantaneously emitted and was collected with a time correlating single photon (TCSPC) workstation to contrast SHG signal to fluorescence signal. We introduced an index (F-SHG) described as the ratio between the long lifetime (typically intrinsic fluorescence) and fast lifetime (SHG) which is obtained by two exponential components fitting. The photons which are provided from SHG signal (fast time slope for a short decay) can be contrasted with a fluorescence signal (slow time slope for a long decay) as encountered for autofluorescence (cell and collagen protein).

As shown in Graph 1, the change in F-SHG index was monitored according to tissue components and confirm identity of the collagen network collected with MacroSHG. The results revealed a precise range of F-SHG index (from 2 to 15), distributed among 4 defined area (I, II, III, IV) in relation with matrix component. For Wharton's jelly, fluorophore (Alexa $488^{\mathrm{TM}}$ ) has been specifically detected by immunolabelling in part I. The range detection of fluorescence is restricted in part II (from 2 to 3.3) concerned more specifically global autofluorescence (AF) coming from matrix tissue, as an indication on the cellular density in matrix and its effect on global autofluorescence. Specific signal for nacre, cartilage and tumor was strictly confined in area II with a range from 3.3 to 4.4. When autofluorescenceis predominant in the presence of SHG (skin, vessel, cartilage, tendon), F-SHG ratio values was included with a range from 5 to 8 (area III). For pure SHG signal relative to matrix collagen, index was significatively well distributed in the last range IV (from 8 to 15).

\section{Discussion}

Here we have integrated multiphotonmultiscale and multimodalities on laser scanningmacroscopy to detect collagen network. In the field of nonlinear optical imaging, we propose in this work an innovative and invasiveless

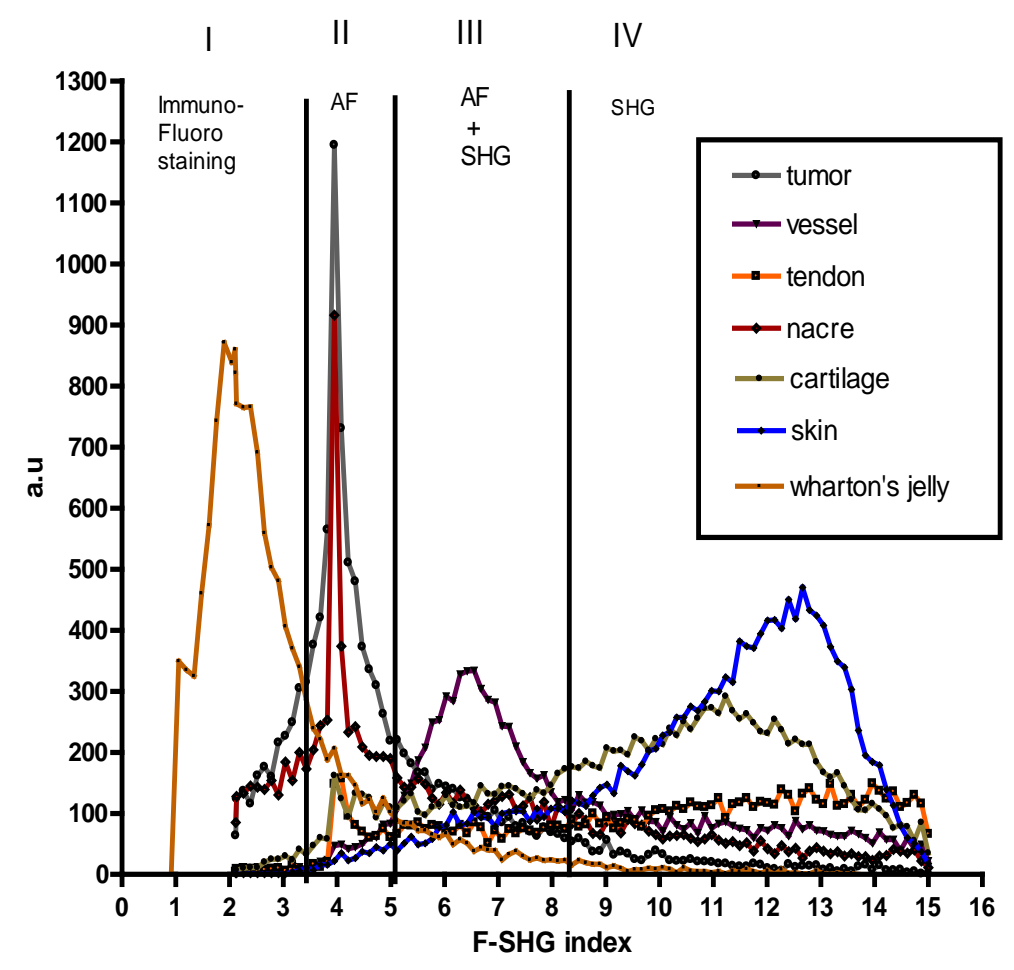

Graph 1. Distribution of F-SHG index is defined in 4 well defined areas (part I, II, III and IV) respectively in close relation with detection of extrinsic fluorescence (I), autofluorescence (II), mixed autofluorescence and SHG (III), and SHG collagen (IV). 
technique imaging (Multiphoton and SHG Macroscope) to collect cartography of SHG signal. Multiphotonmacroscopy allows observing a first fluorescent marker thanks to the 2PEF (range I on F-SHG ratio) and visualizing the collagen without marking with the SHG (range IV). The ability of collagen to be detected as a harmonophore by F-SHG index without the need for an exogenous probe could be a useful tool for bio-diagnosis (range III and IV on F-SHG ratio) or to monitor the state of network collagen prior to clinical implantation. We showed that multiscale detection of second harmonic signal specific to collagen network is a non-invasive and potential technology in tomography to characterize the functional and organised matrix [5] [6]. In conclusion, we have demonstrated with regard to the conventional techniques for the visualization of the collagen that the generation of the harmonious second is a specific sensitive technique of fibrillaires collagens. Finally, it is the only technique which allows reaching the 3D organization of the collagen in tissues. From a general point of view, our studies so demonstrated the relevance of the SHG macroscopy to have a control of the quality of one biomaterial in time with a potential opening in CARS (Coherent Anti-Stokes Raman Scattering) applications [7].

\section{Acknowledgements}

The authors would like to thank Didier Hentsch, and Jean-Luc Vonesh (IGBMC, Strasbourg, France) for their indisensable help with multiphoton imaging. This study was funded in part by grants from CUGN 54, FR3209 CNRS, Région Lorraine, RTmfm MRCT CNRS, GIS IBISA, ARC, GDR 2588 CNRS and Agence National de la Recherche (ANR-05-JC5-51629).

\section{References}

[1] Werkmeister, E., Isla, N.D., Marchal, L., Stoltz, J.F. and Dumas, D. (2008) Interest of Second Harmonic Generation Imaging for Diagnosis in Thick and Opaque Tissue. Biorheology, 45, 375-383.

[2] Bayan, C., Levitt, J.M., Miller, E., Kaplan, D.L. and Georgakoudi, I. (2009) Fully Automated, Quantitative, Noninvasive Assessment of Collagen Fiber Content and Organization in Thick Collagen Gels. Journal of Applied Physics, 105, Article ID: 102042. http://dx.doi.org/10.1063/1.3116626

[3] Dumas, D., Henrionnet, C., Hupont, S., Werkmeister, E., Stoltz, J.F., Pinzano, A. and Gillet, P. (2010) Innovative TCSPC-SHG Microscopy Imaging to Monitor Matrix Collagen Neo-Synthetized in Bioscaffolds. Bio-Medical Materials and Engineering, 20, 183-188.

[4] Dumas, D. and Hupont, S. (2013) Improved Biomedical Imaging with Multiphoton Excitation. WO Patent No. 2012160312 A3.

[5] Werkmeister, E., Isla, N.D., Netter, P., Stoltz, J.F. and Dumas, D. (2010) Collagenous Extracellular Matrix of Cartilage Submitted to Mechanical Forces Studied by Second Harmonic Generation Microscopy. Photochemistry and Photobiology, 86, 302-310. http://dx.doi.org/10.1111/j.1751-1097.2009.00648.x

[6] Dumas, D., Henrionnet, C., Hupont, S., Werkmeister, E., Hentsch, D., Vonesh, J.L., Netter, P., Magdalou, J., Stoltz, J.F., Gillet, P. and Pinzano, A. (2012) New System for Non-Invasive Exploration of Synthesized Collagen Network Inside Mesenchymal Stem Cell Functionalized Biomaterials Dedicated to Clinical Implantation in Cartilage Lesion. Osteoarthritis and Cartilage, 20, 129-130. http://dx.doi.org/10.1016/j.joca.2012.02.168

[7] Evans, C.L., Potma, E.O., Puoris'haag, M., Cote, D., Lin, C. and Xie, X. S. (2005) Chemical Imaging of Tissue in Vivo with Video-Rate-Coherent Anti-Stokes-Raman Scattering (CARS) Microscopy. Proceedings of the National Academy of Sciences, 102, 16807-16812. http://dx.doi.org/10.1073/pnas.0508282102 
Scientific Research Publishing (SCIRP) is one of the largest Open Access journal publishers. It is currently publishing more than 200 open access, online, peer-reviewed journals covering a wide range of academic disciplines. SCIRP serves the worldwide academic communities and contributes to the progress and application of science with its publication.

Other selected journals from SCIRP are listed as below. Submit your manuscript to us via either submit@scirp.org or Online Submission Portal.
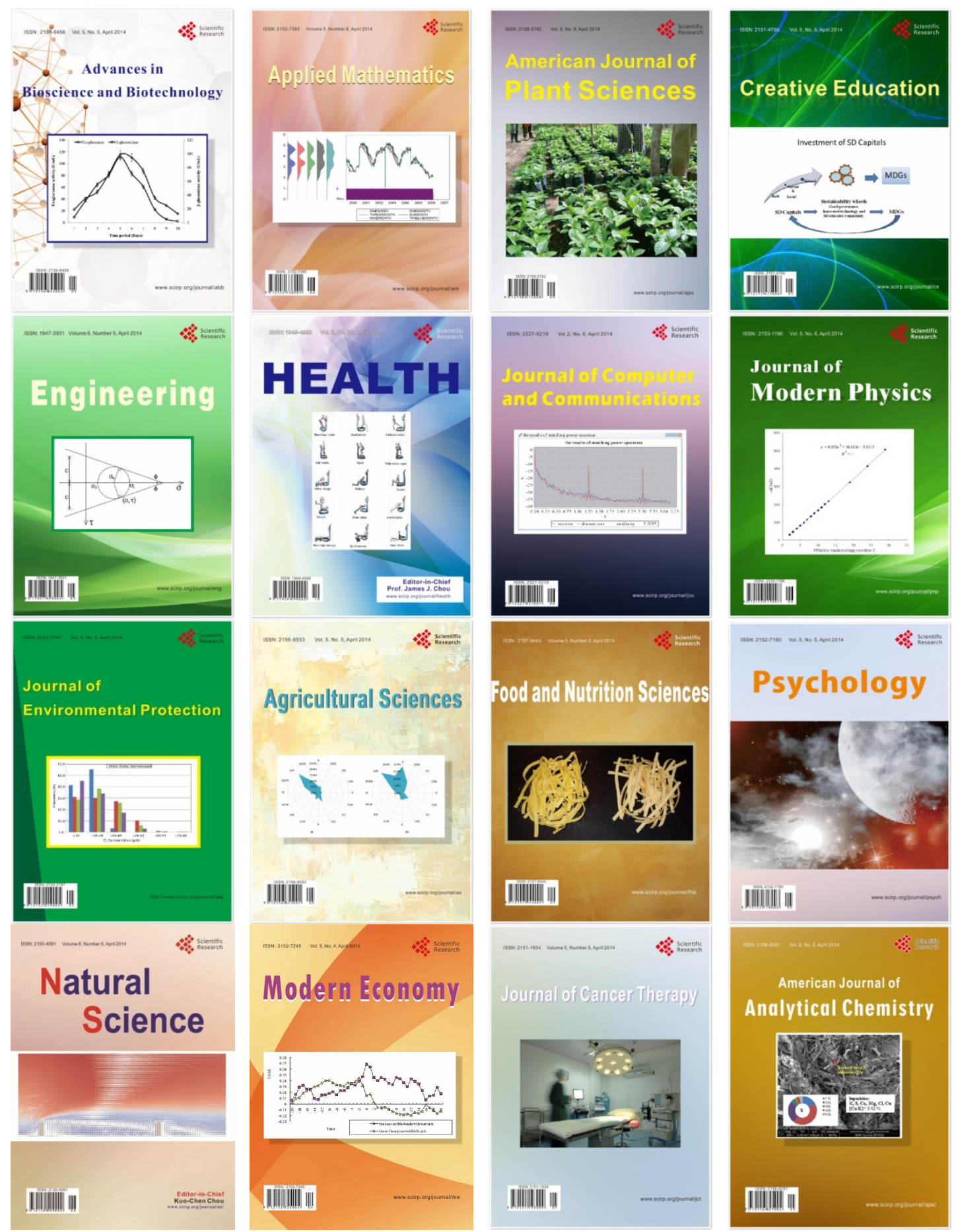